\title{
Establishment of a Predictive Model Related to Pathogen Invasion for Infectious Diseases and Its Diagnostic Value in Fever of Unknown Origin
}

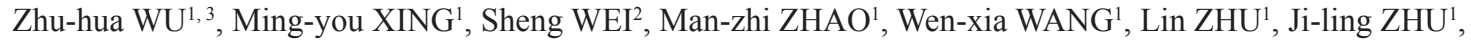 \\ Cai-feng ZHENG ${ }^{1}$, Si-jun WANG ${ }^{1}$, Jun-ying $\mathrm{QI}^{1}$, Jian-xin $\mathrm{SONG}^{1 \#}$ \\ ${ }^{1}$ Department of Infectious Diseases, Tongji Hospital, Tongji Medical College, Huazhong University of Science and Technology, \\ Wuhan 430030, China \\ ${ }^{2}$ Department of Epidemiology and Biostatistics, School of Public Health, Tongji Medical College, Huazhong University of \\ Science and Technology, Wuhan 430030, China \\ ${ }^{3}$ Department of Oncology, the Third Affiliated Hospital of Xinxiang Medical University, Xinxiang 453000, China
}

(C) Huazhong University of Science and Technology 2018

\begin{abstract}
Summary: The present study aimed to establish a list of parameters indicative of pathogen invasion and develop a predictive model to distinguish the etiologies of fever of unknown origin (FUO) into infectious and non-infectious causes. From January 2014 to September 2017, 431 patients with FUO were prospectively enrolled in the study population. This study established a list of 26 variables from the following 4 aspects: host factors, epidemiological factors, behavioral factors, and iatrogenic factors. Predefined predicted variables were included in a multivariate logistic regression analysis to develop a predictive model. The predictive model and the corresponding scoring system were developed using data from the confirmed diagnoses and 9 variables were eventually identified. These factors were incorporated into the predictive model. This model discriminated between infectious and non-infectious causes of FUO with an AUC of 0.72 , sensitivity of 0.71 , and specificity of 0.63 . The predictive model and corresponding scoring system based on factors concerning pathogen invasion appear to be reliable screening tools to discriminate between infectious and non-infectious causes of FUO.
\end{abstract}

Key words: fever of unknown origin; predictive model; etiology; empiric therapy

The classic definition of fever of unknown origin (FUO) was first proposed by Petersdorf and Beeson ${ }^{[1]}$ in 1961. In 1991, Durack and Street ${ }^{[2]}$ modified the definition, and this new definition has been widely used since then. Encompassing both very rare and relatively common entities, over 200 diverse etiologies of FUO have been described ${ }^{[3]}$. Etiological studies demonstrated that infections were among the most common diagnoses ${ }^{[4]}$. Despite great advances in diagnostic tools, such as various serological assays and improved imaging techniques, FUO remains a formidable challenge for clinicians ${ }^{[5]}$.

Several studies have proposed staged diagnostic algorithms to minimize non-focused testing ${ }^{[6]}$. Taking a complete and thorough history is the first step in identifying potential diagnostic clues $^{[7]}$. Obtaining a

Zhu-hua WU, E-mail: 598633905@qq.com

${ }^{\#}$ Correspondence author, E-mail: songsingsjx@sina.com detailed patient history may also provide essential clues about the etiology and is typically preferable to ordering more screening tests. A thorough history includes the onset of symptoms, other signs or symptoms emerging or disappearing during the course of the disease, and any accompanying signs or symptoms $\mathrm{s}^{[8]}$. The process by which pathogens first invade an organism is the unique pathogenesis for infectious diseases, and this is significantly distinct from the pathogenesis of noninfectious diseases. Following pathogen invasion, the immune system is stimulated, particularly the innate and adaptive immune responses. However, to date, there has been a lack of direct and effective methods that may be used to clarify whether or not pathogen invasion occurs before the appearance of symptoms in a patient with FUO.

In accordance with the specific pathogenesis of infectious diseases, the aim of the present study was 
to develop a predictive model based on factors related to pathogen invasion at time of FUO onset in order to promptly and efficiently distinguish between the etiologies of infectious and non-infectious processes.

\section{MATERIALS AND METHODS}

This was a single-institution prospective observational study performed at Tongji Hospital from January 2014 to September 2017. Only patients with classical FUO satisfying the modified criteria of Durack and Street were included, while subjects with immunosuppression, nosocomial FUO, or neutropenic FUO were excluded. FUO was defined as fever lasting more than 3 weeks with a temperature measuring higher than $38.3^{\circ} \mathrm{C}$ on several occasions, and the cause of the fever remains unclear after 1 week of in-hospital evaluation.

In infectious diseases, the most probable and critical time period during which pathogens invade the body is $1-2$ weeks before the emergence of apparent clinical signs and symptoms and this is defined as the onset stage; the latent time of some cases may be longer. Based on the literature and our clinical experience, the present study created a screening list related to medical history (table 1) to search for clues of pathogen invasion; the list included host factors, epidemiological factors, behavioral factors, and iatrogenic factors ${ }^{[8,9]}$. Every item had a standard and strict definition, as shown in supplement 1 . In accordance with the screening list, comprehensive and detailed histories were obtained from enrolled patients on the first day of admission, in order to evaluate the possibility and route of pathogen invasion at the onset stage of disease. Two internists collected and interpreted patients' data, and they were both blinded to the final diagnoses.

Final diagnoses were determined by a committee composed of three specialist physicians (with experience in infectious disease, malignancies, and autoimmune diseases), a radiologist, and a laboratory specialist in cases of specific diseases. Patients were classified into two categories: infectious or noninfectious. Definite infections were diagnosed by means of the following results plus imaging if necessary:

Table 1 The screening list of clues related to the medical history of pathogen invasion

\section{Host factors}

Injury of mucosal barrier

(1) Injured skin

Pedicure, acupuncture, cupping, tattoo, open wounds, dermatopathy

(2) Injured oral mucosa

Oral ulcers

Diseases associated with teeth and gums

Pharyngitis

(3) Injured mucosa of the respiratory tract

Rhinitis

Upper respiratory tract infection

Bed-ridden

Bronchitis

Bronchiectasia

(4) Injured mucosa of the gastrointestinal tract

Gastritis

Peptic ulcer

Diarrhea

Cholangitis

Pancreatitis

Appendicitis

(5) Injured mucosa of urinary tract

Cystitis

Pyelonephritis

(6) Injured genital tract

Genital tract infection

Unclean sexual contact

Chronic underlying diseases

(1) Diabetes

(2) Chronic liver disease

(3) Chronic kidney disease

History of amygdalectomy or splenectomy

Malnutrition

Hereditary diseases

\section{Epidemiological factors}

Environmental pollution of living or working conditions Feeding and close contact with animals carrying the pathogen Intimate contact with people living in epidemic areas

Living in epidemic areas

\section{Behavioral factors}

Sudden changes in the rhythm of life or work

Presence of a cold

Long-term work overload

Chronic sleep deficit

Poor eating habits

Iatrogenic factors

Toothwash or dental filling

Cosmetology

Induced abortion

Artificial implantation of foreign bodies

Artifistulation 
positive body fluid culture; positive serology result; and positive polymerase chain reaction and immune assay for a pathogenic organism ${ }^{[10]}$. Non-infections, including malignancies, autoimmune diseases, and miscellaneous causes, were diagnosed according to the corresponding diagnostic criteria. The follow-up period to obtain the definitive diagnoses of enrolled patients was 6 months.

\subsection{Statistical Analysis}

Categorical data were presented as number and percentages and were analyzed using the chi-square test or Fisher's exact test to compare the results of patients in the infectious disease and non-infectious disease subgroups. The polynomial predictive model was constructed using a logistic regression model including all predictor variables. Akaike's information criterion (AIC) was used to judge the fitness of the models. The reduction in AIC between models was evaluated by the likelihood ratio test ${ }^{[11]}$. The final predictive model was identified as having the significantly lowest AIC value. The difference was considered statistically significant at $P<0.05$. The receiver operating characteristic (ROC) curve was obtained by plotting sensitivity against 1 -specificity at each cut-off value. Diagnostic accuracy was assessed by the area under the curve. All analyses were performed using SPSS statistical software, version 18.0 (SPSS, USA) and Empower Stats (http:// www.empowerstats.com).

\section{RESULTS}

\subsection{Distribution of Etiologies}

There were 697 febrile adults during the nearly 4-year period at the hospital. Of these, 266 patients were excluded for the following reasons: not meeting the basic criteria of FUO (40.5\%); discharge from the hospital within 5 days (20.4\%); and the diagnosis was not obtained within 6 months after discharge $(8.7 \%)$ or patients were lost to follow up (30.4\%). The remaining 431 patients that fulfilled all the study criteria were eventually included in the analysis. Among them, infectious disease was the most common cause of FUO. Furthermore, various antibiotics were administered to a high percentage of patients prior to confirmation of an exact diagnosis. In order of proportion, the most prevalent FUO causes were tuberculosis, sepsis, brucellosis, malignant lymphoma and adult-onset Still disease as shown in table 2.

Table 2 Causes of fever in FUO patients

\begin{tabular}{lrlr}
\hline Infectious causes & $n(\%)$ & Non-infectious causes & $n(\%)$ \\
\hline Bacterial & $195(45.2)$ & Autoimmune diseases & $93(21.6)$ \\
Tuberculosis & $49(11.4)$ & Adult-onset Still disease & $29(6.7)$ \\
Brucellosis & $38(8.8)$ & Undifferentiated connective tissue disease & $15(3.5)$ \\
Sepsis & $41(9.5)$ & Systemic lupus erythematosus & $14(3.2)$ \\
Pulmonary infection & $21(4.9)$ & Vasculitis & $10(2.3)$ \\
Urinary infection & $6(1.4)$ & Sicca syndrome & $3(0.7)$ \\
Endocarditis & $4(0.9)$ & Others & $22(5.1)$ \\
Typhoid fever & $3(0.7)$ & Neoplasms & $62(14.4)$ \\
Abscess & $10(2.3)$ & Malignant lymphoma & $35(8.1)$ \\
Others & $23(5.3)$ & Leukemia & $10(2.3)$ \\
Viral & $24(5.6)$ & Multiple myeloma & $2(0.5)$ \\
Epstein-Barr virus & $14(3.2)$ & Cervical cancer & $3(0.7)$ \\
Cytomegalovirus & $2(0.5)$ & Prostatic cancer & $2(0.5)$ \\
Virus meningitis & $3(0.7)$ & Glandular cancer & $2(0.5)$ \\
Others & $5(1.2)$ & Others & $8(1.9)$ \\
Fungal & $12(2.8)$ & Miscellaneous & $35(8.1)$ \\
Pulmonary fungal infection & $6(1.4)$ & Kikuchi’s syndrome & $15(3.5)$ \\
Fungal meningitis & $1(0.2)$ & Subacute thyroiditis & $9(2.1)$ \\
Others & $5(1.2)$ & Others & $11(2.6)$ \\
Mycoplasma & $4(0.9)$ & &
\end{tabular}

\subsection{Medical History Characteristics}

Table 3 shows the results of the univariate analysis of certain invasive clues. There was no significant difference in age between the infectious and noninfectious groups. The male patients in the infectious group were significantly more than those in the non- infectious group. Feeding and close contact with animals carrying pathogen and presence of a cold were significantly more common in the infectious group than in the non-infectious group $(P<0.05)$, and these were all determined to be risk factors for infectious diseases. Univariate analysis showed no statistical 
difference between the two groups in the factors of injury of the mucosal barrier, chronic underlying diseases, malnutrition, environmental pollution of living or working conditions, intimate contact with people living in the epidemic areas, sudden changes in the rhythm of life or work, iatrogenic factors, chronic sleep deficit, poor eating habits and long-term work overload. In the present study, intimate contact with people living in epidemic areas, poor eating habits and malnutrition were only identified in the infectious group, with $6 \%, 4 \%$ and $2 \%$ respectively. The history of amygdalectomy or splenectomy was only identified in the non-infectious group, with $2.6 \%$. The remaining variables were not found in either group.

\subsection{Performance of the Predictive Model}

Based on the results of the univariate analysis, a multinomial logistic regression analysis of all predefined variables was conducted to develop a predictive model consisting of 5 variables, including sex, injury of the mucosal barrier, feeding or close contact with animals carrying pathogen, presence of a cold, and iatrogenic factors. These factors were all positive indicators of infectious causes with odds ratios greater than one. The details of the predictive model are provided in table 4 . By rounding the coefficients of each variable in the model, the score was predicted as presented in table 4 . The best cut-off point was 15 .

Table 3 General characteristics of infection and non-infection

\begin{tabular}{|c|c|c|c|}
\hline General characteristics & Infection & Non-infection & $P$ value \\
\hline Age, years & $46.96 \pm 16.88$ & $45.11 \pm 17.09$ & 0.233 \\
\hline Sex, male $(\%)$ & $148(61.4)$ & $90(47.4)$ & 0.004 \\
\hline Injury of mucosal barrier $(\%)$ & $85(35.3)$ & $52(27.4)$ & 0.080 \\
\hline Chronic underlying diseases $(\%)$ & $28(11.6)$ & $18(9.5)$ & 0.474 \\
\hline Malnutrition $(\%)$ & $2(0.8)$ & $2(1.1)$ & 1.000 \\
\hline Environmental pollution of living or working conditions (\%) & $18(7.5)$ & $7(3.7)$ & 0.095 \\
\hline Feeding and close contact with animals carrying pathogen $(\%)$ & $35(14.5)$ & $2(1.1)$ & $<0.001$ \\
\hline Intimate contact with people living in the epidemic areas (\%) & $5(2.1)$ & $2(1.1)$ & 0.472 \\
\hline Sudden changes in the rhythm of life or work (\%) & $9(3.7)$ & $8(4.2)$ & 0.808 \\
\hline Presence of a cold $(\%)$ & $54(22.4)$ & $22(11.6)$ & 0.003 \\
\hline Long-term work overload (\%) & $12(5.0)$ & $5(2.6)$ & 0.319 \\
\hline Chronic sleep deficit (\%) & $16(6.6)$ & $16(8.4)$ & 0.484 \\
\hline Poor eating habits $(\%)$ & $1(0.4)$ & $1(0.5)$ & 1.000 \\
\hline Iatrogenic factors $(\%)$ & $14(5.8)$ & $5(2.6)$ & 0.155 \\
\hline
\end{tabular}

Table 4 Multiple regression analysis for diagnosis of infection in FUO patients

\begin{tabular}{lccccc}
\hline Variable & $\beta$-Coefficient & Standard error & $P$ value & OR (95\%CI) & Score \\
\hline Sex & 0.61 & 0.24 & 0.011 & $1.83(1.15-2.92)$ & 6 \\
Injury of the mucosal barrier & 0.50 & 0.23 & 0.031 & $1.65(1.05-2.60)$ & 5 \\
Feeding and close contact with animals carrying pathogen & 2.98 & 0.75 & $<0.001$ & $19.71(4.57-85.05)$ & 30 \\
Presence of a cold & 1.12 & 0.29 & $<0.001$ & $3.05(1.71-5.44)$ & 11 \\
Iatrogenic factors & 1.18 & 0.56 & 0.034 & $3.26(1.10-9.70)$ & 12 \\
\hline OR:
\end{tabular}

OR: odds ratio; $95 \%$ CI: 95\% confidence intervals

This predictive model successfully identified infectious causes of FUO. The performance of this scoring system was evaluated by means of a ROC curve (fig. 1). The area under the curve was $0.72(95 \%$ confidence interval, 0.67 to 0.78 ), and the sensitivity and specificity were 0.71 and 0.63 , respectively. The positive likelihood ratio was 1.92 , the negative likelihood ratio was 0.46 , and the positive and negative predicted values were 0.71 and 0.63 , respectively.

\section{DISCUSSION}

The etiologies of FUO are complicated, while comprehensive history collection and complete

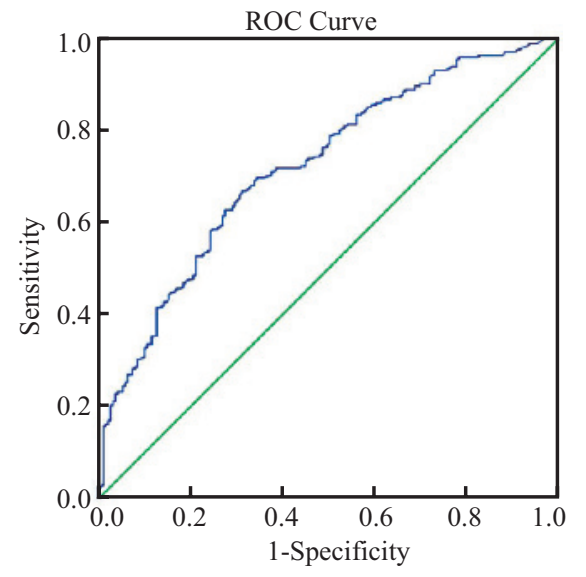

Fig. 1 Receiver-operating characteristic curves of the predict model 
physical examination are important prerequisites and bases for diagnosis ${ }^{[8]}$. The primary evaluation of a patient with FUO should begin with taking a detailed and thorough history ${ }^{[7]}$. However, most clinicians ignore the critical onset stage, including the incubation period before early clinical symptoms appear and the initial pathogenesis preceding the occurrence of these symptoms. The reason for this may be that they simply evaluate only common causes or only focuse on current clinical manifestations. In this study, the clinical focus was on the initial onset stage, i.e., 1-2 weeks prior to the appearance of obvious symptoms and signs. It should be noted that the duration of the onset stage may be longer in certain diseases. Several previous studies have focused on developing a procedure or tool, mainly based on laboratory markers, to discriminate between infectious diseases and non-infectious diseases in patients with $\mathrm{FUO}^{[9,12,13]}$. Moreover, in contrast with non-infectious diseases, infectious diseases undergo the process of pathogen invasion during the onset stage in addition to inducing immune responses ${ }^{[14]}$. At present, due to a complex history and shortage of innovative ideas, few reports have emphasized the essentiality of clues related to pathogen invasion in the pathogenesis of infectious diseases during the onset stage. Therefore, in our study design, we sought to identify clues related to pathogen invasion at the initial onset stage, in order to distinguish infectious diseases from non-infectious diseases as the causes of FUO.

The pathogenesis of infectious diseases is closely related to the interaction of pathogens, host immunity, and environmental factors ${ }^{[14]}$. As the first barrier of the body to resist the invasion of internal or external pathogens, the mucosal barrier system is a fundamental part of the innate immune system ${ }^{[15-17]}$. Patients with the presence of a cold, which can lead to immune dysfunction, are susceptible to infection. Patients in close contact with certain animals or those who live in endemic areas may suffer from zoonotic infection ${ }^{[18]}$. In addition, aggressive medical examinations, especially improper medical manipulations, increase the risk of infection $^{[19-21]}$. Accordingly, the screening list of clues to identify pathogen invasion was comprehensively developed from the four aspects of host factors, epidemiological factors, behavioral factors, and iatrogenic factors.

There are particular strengths in this study. First, by analyzing the systematic and complete list, physicians can avoid missing valuable information during clinical history-taking. Second, each clue to pathogen invasion is defined in detail, so that first-line clinicians are able to comprehend the implication of pathogen invasion and analyze its probabilities. Detailed definitions can greatly reduce the interference of subjective factors. Third, the score model, which is easily accessible and may be widely used in clinical practice, provides a quantitative analysis of the complicated disease history. Fourth, combined with classical strategy guided by potentially diagnostic clues $^{[22]}$, the score model can determine the diagnosis of infectious diseases upon initial judgment and then minimize nonfocused testing. Finally, infectious diseases are still the main cause of $\mathrm{FUO}^{[23]}$, especially in developing countries. A great challenge for clinicians is that highstrength, over-broad antibiotics are often administered to patients with FUO before any evidence of infection has been identified ${ }^{[24]}$. The causes of FUO could be broadly separated into infection or non-infection using the score model. Although some scholars believe that it is inappropriate to prescribe antibiotics for FUO before a diagnosis is confirmed, the positive clues of pathogen invasion can assist the physician in making an accurate decision about whether or not to prescribe antibiotics. In light of certain clues, the possible routes of invasion, such as the skin, respiratory tract, gastrointestinal tract, and urinary tract, can be identified. Based on this, it is beneficial for physicians to make wiser choices regarding the use of antibiotics and make empirical treatment more reasonable.

This study also has some limitations. First, the results of the score model could not provide information related to identifying the definite etiology of infectious diseases. Second, compared to previous reports in the literature, the proportion of infectious diseases was higher in this study. Infectious diseases still represent a major component of the FUO etiologies in developing countries. Due to the limitation of the sample size, the study did not include a validation cohort to verify the diagnostic value of the score model.

An innovative strategy known as the "twostep" diagnostic algorithm was proposed to rapidly discriminate the etiology of FUO as infectious or non-infectious, and it has been registered on http:// www.clinical trials.gov in 2013 under the registration number NCT02035670. With this method, the first step is to discover and analyze the possibility of pathogen invasion at the onset phase, as demonstrated in the present study. The second step, which is specific to the phase of disease progression, takes into consideration gender, clinical symptoms and signs, and laboratory examination results. Future research concerning combined application of this two-step diagnostic algorithm will enable improvement of the sensitivity and specificity of distinguishing FUO etiology as infectious or non-infectious and enable clinicians to more distinctly determine whether empirical use of antibiotics is necessary. Some experts believe that belated application of antibiotics will make diseases delayed or more serious, if the final diagnosis is certain infectious diseases ${ }^{[25-27]}$. This diagnostic algorithm can also provide a tentative diagnosis to guide early indepth treatment of non-infectious diseases, particularly 
certain malignancies or autoimmune diseases that may progress quickly.

In conclusion, the innovative score model comprising clues of pathogen invasion, including host factors, epidemiological factors, behavioral factors, and iatrogenic factors, is a valuable tool that may be used to rapidly identify the cause of infection in patients with FUO. As a result, this model can assist clinicians with subsequent targeted examinations and reasonable selection of antibiotics.

\section{Acknowledgement}

We would like to thank all the physicians at the hospitals around the country for their participation and data collection. This project would not have been possible without their contributions.

\section{Conflict of Interest Statement}

The authors declare that there is no conflict of interest.

\section{REFERENCES}

1 Petersdorf RG, Beeson PB. Fever of unexplained origin: report on 100 cases. Medicine (Baltimore), 1961,40:130

2 Durack DT, Lukes AS, Bright DK. New criteria for diagnosis of infective endocarditis: utilization of specific echocardiographic findings. Duke Endocarditis Service. Am J Med, 1994,96(3):200-209

3 Horowitz HW. Fever of unknown origin or fever of too many origins? N Engl J Med, 2013,368(3):197-199

$4 \mathrm{Hu} \mathrm{Y,} \mathrm{Lu} \mathrm{H,} \mathrm{Zhang} \mathrm{Y,} \mathrm{et} \mathrm{al.} \mathrm{Fever} \mathrm{of} \mathrm{unknown} \mathrm{origin:}$ revisit of 142 cases in a tertiary Chinese hospital. Biosci Trends, 2008,2(1):44-46

5 Vanderschueren $\mathrm{S}$, Knockaert D, Adriaenssens $\mathrm{T}$, et al. From prolonged febrile illness to fever of unknown origin: the challenge continues. Arch Intern Med, 2003,163(9):1033-1041

6 Cunha BA. Fever of unknown origin: focused diagnostic approach based on clinical clues from the history, physical examination, and laboratory tests. Infect Dis Clin North Am, 2007,21(4):1137-1187

7 Bleeker-Rovers CP, Vos FJ, de Kleijn EM, et al. A prospective multicenter study on fever of unknown origin: the yield of a structured diagnostic protocol. Medicine (Baltimore), 2007,86(1):26-38

8 Kaya A, Ergul N, Kaya SY, et al. The management and the diagnosis of fever of unknown origin. Expert Rev Anti Infect Ther, 2013,11(8):805-815

9 Efstathiou SP, Pefanis AV, Tsiakou AG, et al. Fever of unknown origin: discrimination between infectious and non-infectious causes. Eur J Intern Med, 2010,21(2):137143

10 Arvaniti V, D'Amico G, Fede G, et al. Infections in patients with cirrhosis increase mortality fourfold and should be used in determining prognosis. Gastroenterology, 2010,139(4):1246-1256

11 Huelsenbeck JP, Hillis DM, Nielsen R. A likelihoodratio test of monophyly. Syst Biol, 1996,45(4):546-558

12 Kim SE, Kim UJ, Jang MO, et al. Diagnostic use of serum ferritin levels to differentiate infectious and noninfectious diseases in patients with fever of unknown origin. Dis Markers, 2013,34(3):211-218

13 Li L, Zhang JZ, Zhao MZ, et al. Applied value of monitoring serum hepcidin in differential diagnosis of infection versus tumor fevers. J Huazhong Univ Sci Technol Med Sci, 2017,37(2):253-256

14 Si-Tahar M, Touqui L, Chignard M. Innate immunity and inflammation--two facets of the same anti-infectious reaction. Clin Exp Immunol, 2009,156(2):194-198

15 Akgul Y, Word RA, Ensign LM, et al. Hyaluronan in cervical epithelia protects against infection-mediated preterm birth. J Clin Invest, 2014,124(12):5481-5489

$16 \mathrm{Yu} \mathrm{Q}$, Yuan L, Deng J, et al. Lactobacillus protects the integrity of intestinal epithelial barrier damaged by pathogenic bacteria. Front Cell Infect Microbiol, 2015,5:26

17 Yamaguchi T, Yamada H. Role of mechanical injury on airway surface in the pathogenesis of Pseudomonas aeruginosa. Am Rev Respir Dis, 1991,144(5):11471152

18 Al Dahouk S, Nockler K, Hensel A, et al. Human brucellosis in a nonendemic country: a report from Germany, 2002 and 2003. Eur J Clin Microbiol Infect Dis, 2005,24(7):450-456

19 Deshpande A, Smith GW, Smith AJ. Biofouling of surgical power tools during routine use. J Hosp Infect, 2015,90(3):179-185

20 Maugars Y, Albert JD, Bard H, et al. Prevention of iatrogenic infections in interventional rheumatology: Optimal measures but adapted to each risk. Joint Bone Spine, 2016,83(3):250-253

21 Higa JT, Gluck M, Ross AS. Duodenoscope-Associated Bacterial Infections: A Review and Update. Curr Treat Options Gastroenterol, 2016,14(2):185-193

22 Mulders-Manders C, Simon A, Bleeker-Rovers C. Fever of unknown origin. Clin Med (Lond), 2015,15(3):280284

23 Ergonul O, Willke A, Azap A, et al. Revised definition of 'fever of unknown origin': limitations and opportunities. J Infect, 2005,50(1):1-5

24 Bryan CS, Ahuja D. Fever of unknown origin: is there a role for empiric therapy? Infect Dis Clin North Am, 2007,21(4):1213-1220

25 Berjohn CM, Fishman NO, Joffe MM, et al. Treatment and Outcomes for Patients with Bacteremic Pneumococcal Pneumonia. Medicine (Baltimore), 2008,87(3):160-166

26 Cinel I, Dellinger RP. Advances in pathogenesis and management of sepsis. Curr Opin Infect Dis, 2007,20(4):345-352

27 Clec'h C,Timsit JF, De Lassence A, et al. Efficacy of adequate early antibiotic therapy in ventilator-associated pneumonia: influence of disease severity. Intensive Care Med, 2004,30(7):1327-1333

(Received July 12, 2018; revised Oct. 23, 2018)

\section{Supplement 1}

\section{Host factors}

1. Injury of mucosal barrier

1.1 Injured skin

Pedicure, acupuncture, cupping, tattoo, open wounds, dermatopathy: contamination of the incision, massive bleeding, red swelling with pain, ulceration, and purulent secretion. 
1.2 Injured oral mucosa

Oral ulcers: large area, multiple sites, and recurrent episodes.

Diseases associated with teeth and gums: gingivitis, periodontitis, pulpitis, pericoronitis, and purulent gums.

Pharyngitis: pharyngeal sensation of a foreign body with pain and itch, sputum cannot be egested or swallowed, and nausea or dysemesia when brushing teeth.

1.3 Injured mucosa of the respiratory tract

Rhinitis: nasal congestion, increased secretions, accompanying headache, hyposmia, nasal twang, insomnia, or imaging diagnosis.

Upper respiratory tract infection: nasal obstruction, nasal discharge, pharyngodynia, cough, and expectoration.

Bed-ridden: gastroesophageal reflux and irritating cough.

Bronchitis: cough, expectoration, and asthma.

Bronchiectasis: chronic cough, purulent sputum, and recurrent hemoptysis.

1.4 Injured mucosa of the gastrointestinal tract

Gastritis: postprandial epigastric discomfort, recurrent epigastric pain, belching, acid reflux, vomiting, or recent gastroscopy diagnosis.

Peptic ulcer: abdominal pain, hematemesis, hematochezia, or digestive endoscopy diagnosis.

Diarrhea: abdominal pain and changes in stool frequency and/or form appearance.

Cholangitis: abdominal pain, jaundice, epigastric discomfort, and imaging diagnosis.

Pancreatitis, appendicitis: corresponding diagnostic criteria.

1.5 Injured mucosa of the urogenital tract

Cystitis, pyelonephritis: frequency of micturition, urgency of urination, urodynia, hematuria, pyuria, and urinary retention.

1.6 Injured mucosa of the reproductive tract

Genital tract infection: burning sensation, festering, abnormal increase of secretion, and abnormal odor.

1.7 Unclean sexual contact

2. Chronic underlying diseases

2.1 Diabetes

2.2 Chronic liver disease

2.3 Chronic kidney disease

3. History of amygdalectomy or splenectomy

4. Malnutrition

Malnutrition: insufficiency of nourishment, obvious marasmus, weakness, and disappearance of subcutaneous fat.

5. Hereditary diseases

Hereditary diseases: hereditary hemolytic anemia, Wilson disease, and other hereditary diseases causing damage to liver function, kidney function, and immune function.

\section{Epidemiological factors}

1. Environmental pollution of living or working conditions

Air, water, soil, and food contaminated by pathogenic microorganisms; living in a closed and humid environment for a long time.

2. Feeding and close contact with animals carrying the pathogen

3. Intimate contact with people living in epidemic areas

4. Living in epidemic areas

\section{Behavioral factors}

1. Sudden changes in the rhythm of life or work

Changes in the habits of daily life, such as changes in living, eating, dressing, etc.; recent work has made patient feel more tired and stressed, workload has increased significantly so that the hours of sleeping or rest have decreased.

2. Presence of a cold

A sudden change in weather; catching a chill; exposure to rain; feeling frozen in one's sleep; eating a lot of cold food.

3. Long-term work overload

The daily workload is far beyond the limits of the patient's physical strength.

4. Chronic sleep deficit

Sleeping less than 4-6 hours a day or poor sleep quality.

5. Poor eating habits

Unbalanced intake of nutrients, often ingestion of undercooked food or frequent gluttony.

\section{Iatrogenic factors}

1. Toothwash or dental filling

2. Cosmetology

3. Induced abortion

4. Artificial implantation of foreign bodies

5. Artifistulation 